Artigo Original

Gestão em Saúde

\title{
CONHECIMENTO DE GESTANTES DE UMA UNIDADE DE SAÚDE SOBRE OS CUIDADOS COM O RECÉM-NASCIDO
}

\section{Knowledge of pregnant women of a Health Unit on caring for the newborn}

\section{Ernandes Gonçalves Dias}

Enfermeiro. Especialista em Enfermagem do Trabalho e Docência na Saúde. Mestrando em Tecnologia e Inovação em Enfermagem na EERP-USP. Docente na Faculdade Verde Norte (FAVENORTE). E-mail: nandesenf@usp.br

Endereço: Rua Maria Alves da Silva, 58, Icaraí, Monte Azul-MG. CEP: 39500-000.

\section{Cândida Carolina Mendes Novaes}

Graduanda em Enfermagem. Faculdade Presidente Antônio Carlos de Porteirinha.

\section{Ilza Reis dos Santos}

Graduanda em Enfermagem. Faculdade Presidente Antônio Carlos de Porteirinha.

\section{Silvaneide Xavier da Silva}

Graduanda em Enfermagem. Faculdade Presidente Antônio Carlos de Porteirinha.

\section{Janine Cinara Silveira Alves}

Professora Mestre em Ensino de Biologia. Docente na Faculdade Verde Norte (FAVENORTE). 


\section{Conhecimento de gestantes de uma Unidade de Saúde sobre os cuidados com o recém-nascido}

\section{Knowledge of pregnant women of a Health Unit on caring for the newborn}

\section{Resumo}

Objetivo: verificar o conhecimento das gestantes usuárias da Unidade de Saúde Santo Antônio, sobre os cuidados com o recém-nascido. Métodos: Trata-se de uma pesquisa descritiva de natureza quanti-qualitativa, realizada com 17 gestantes. Os dados foram coletados entre março e abril de 2015, através de uma entrevista e foram tratados usando as técnicas de percentagem, desvio padrão (quantitativos) e saturação do discurso (qualitativos). Resultados: Identificou-se prevalência de gestantes na faixa etária entre 23 e 27 anos, casadas, primigestas no $3^{\circ}$ trimestre e baixa escolaridade. Sobre os cuidados com o recém-nascido, $88,2 \% \pm 8,1$ relataram ser necessário amamentar em livre demanda, $82,4 \% \pm 7,8$ não considera necessário complementar a amamentação, $52,9 \% \pm 3,2$ acredita que o banho do bebê deve ser iniciado pela cabeça seguida pelo rosto e corpo, para $58,8 \% \pm 3,8$ a limpeza do coto umbilical deve ser feita somente após o banho e $52,9 \% \pm 3,2$ acreditam que o bebê deve ser colocado para dormir em decúbito dorsal. A maioria não sabe como e quando deve realizar a higiene oral do bebê e acreditam que a posição correta para o bebê arrotar é a vertical, apoiado sobre o ombro. Conclusão: As gestantes têm conhecimento limitado acerca dos cuidados com o recém-nascido, o que pode comprometer o desenvolvimento saudável da criança.

Descritores: Conhecimento; Gestantes; Recém-nascido.

\section{Abstract}

Objective: The care of the newborn involves a lot of responsibility because it is a fragile being who needs comprehensive care. The objective was verify the knowledge of pregnant women users the Santo Antonio Health Unit, on the care of 
the newborn. Method: It is a descriptive of quantitative and qualitative nature, carried out with 17 pregnant women. Data were collected between March and April 2015, through an interview and were treated using the techniques of percentage, standard deviation (quantitative) and saturation of speech (qualitative). Results: He identified himself prevalence of pregnant women in the age group between 23 and 27 years old, married, first pregnancy in the 3rd quarter and low education. On caring for the newborn, $88.2 \pm 8.1 \%$ reported, need of breastfeed on free demand, $82.4 \% \pm 7.8$ does not consider necessary breastfeeding complement, $52.9 \% \pm 3.2$ believes what baby bath should be initiated by the head followed by the face and body, to $58.8 \% \pm$ 3.8 the cleaning the umbilical stump should be made only after the bath and $52.9 \pm$ $3.2 \%$ believe that the baby should be placed to sleep supine. Most do not know how and when should accomplish oral hygiene and baby believe the correct position for the baby burp is vertical, supported about the shoulder. Conclusion: The pregnant women have limited knowledge about the care with the newborn, which can compromise the child's healthy development.

Keywords: Knowledge; Pregnant Women; Infant, Newborn.

\section{INTRODUÇÃO}

No contexto da hierarquização dos serviços de saúde pública no Brasil, a Unidade de Saúde se destaca como a porta de entrada preferencial da gestante no sistema de saúde ${ }^{1}$. O acolhimento pré-natal, realizado nestas Unidades é visto como fator determinante para o acompanhamento adequado à gestante. Cabe à equipe de saúde, criar vínculo com a mulher e buscar compreender os múltiplos significados da gestação para si, sua família e seu parceiro².

O acompanhamento da gestante na Atenção Básica deve contar com profissionais de saúde capazes de atuar de forma integral no cuidado da mulher e do seu filho, com disposição para fornecer 0 apoio necessário durante a gestação ${ }^{3}$.

Durante 0 atendimento pré-natal o profissional deve procurar trabalhar a promoção da saúde de forma a passar informações relevantes para o processo 
gestacional, esclarecer dúvidas e incentivar a gestante quanto às mudanças de hábitos, para diminuir o risco de complicações na gestação ${ }^{4}$.

Ainda na assistência pré-natal, os profissionais devem repassar à gestante orientações sobre os cuidados de higiene, benefícios do exercício físico na gestação, necessidades nutricionais, modificações corporais e emocionais, como também orientações e incentivo ao parto normal e aleitamento materno, cuidados no pós-parto, cuidados com o Recém-Nascido (RN), entre outros ${ }^{5}$.

$\mathrm{O}$ cuidado adequado ao $\mathrm{RN}$ tem sido um dos desafios para reduzir os índices de mortalidade infantil no Brasil. Neste sentido, a redução da mortalidade neonatal implica uma atenção qualificada e segura por parte dos profissionais de saúde, desde o período gestacional até os cuidados destinados aos RN1.

Assim, a educação em saúde durante o pré-natal tem grande importância, pois é neste momento que as gestantes podem tirar suas dúvidas, trocar experiências, conversar sobre assuntos importantes da gestação e cuidados que envolvem tanto a saúde materna quanto a saúde da criança²

A maioria das dificuldades enfrentadas pelas mães, com o RN, é passível de ações preventivas ${ }^{6}$. Frente às adversidades do cuidado com o RN, às gestantes $e$ puérperas necessitam receber orientação, supervisão e esclarecimento de dúvidas sobre os cuidados com seu filho, como para a troca de fralda, banho, cuidados com o coto umbilical e na amamentação ${ }^{7}$.

Devido à vulnerabilidade do RN, muitas mães têm dúvidas e insegurança sobre como cuidar adequadamente de seus filhos. Neste sentido, o cuidado materno é uma atividade que requer conhecimento, pois o medo associado ao despreparo pode levar a falha no cuidado do $\mathrm{RN}^{8}$.

Frente a essas considerações, o estudo teve como objetivo verificar o conhecimento das gestantes usuárias da Unidade de Saúde Santo Antônio do município de Riacho dos Machados-MG, sobre os cuidados com o Recém-nascido.

\section{MÉTODO}


O estudo caracteriza-se como descritivo de natureza quanti-qualitativa, realizado na Unidade de Saúde Santo Antônio do município de Riacho dos Machados, norte de Minas Gerais.

Participaram do estudo 17 das gestantes, com idade igual e maior que 18 anos, usuárias da Unidade de Saúde supracitada, que concordaram em participar do estudo assinando o Termo de Consentimento Livre e Esclarecido, conforme recomenda a Resolução 466/2012 que trata das pesquisas envolvendo seres humanos, independente do período gestacional.

As gestantes foram identificadas a partir de consulta ao cadastro Sistema de Acompanhamento do Programa de Humanização no Pré-Natal e Nascimento, em seguida foi realizada a seleção através das Fichas $A$, onde constava seus respectivos endereços.

Para a obtenção dos dados foi utilizado um roteiro de entrevista estruturada. Os dados foram coletados pelos próprios pesquisadores no período de março a abril de 2015, nas residências das gestantes, no período diurno, em dia e horário previamente agendados. A entrevista foi gravada em áudio mediante a autorização da entrevistada.

Os discursos foram transcritos na íntegra com o objetivo de respeitar o pensamento e as opiniões de cada gestante, na sequência foram realizadas leituras sucessivas dos depoimentos buscando agrupar os dados semelhantes entre si e analisados mediante Análise do Discurso de Bardin (1977).

Os dados quantitativos foram organizados, digitados e processados em computador, utilizando-se de uma planilha do Microsoft Excel 2010 e analisados através do método de epidemiologia descritiva. Optou-se pela elaboração de tabelas para facilitar a compreensão dos dados levantados.

O Comitê de Ética em Pesquisa da Universidade Presidente Antônio Carlos aprovou o projeto de pesquisa pelo Parecer Consubstanciado ㄲo 1411906.

\section{RESULTADOS E DISCUSSÃO}

As gestantes foram caracterizadas quanto à idade, cor, estado civil, escolaridade, moradia, ocupação, renda familiar, período gestacional e número de 
partos. Identificou-se prevalência de gestantes na faixa etária entre 23 e 27 anos (09: 52,9\%), casadas (13: 76,5\%) e pardas (autodefinição) (11: 64,7\%). Quanto ao nível de instrução observou-se baixa escolaridade entre as entrevistadas, 14 $(82,4 \%)$ gestantes tinham até o ensino médio incompleto. Moram em casa própria (04: $23,5 \%)$ e nenhuma exercia ocupação remunerada.

Em relação à idade gestacional, $06(35,3 \%)$ gestante estavam no segundo trimestre, outras $06(35,3 \%)$ no terceiro trimestre e $05(29,4 \%)$ no primeiro trimestre da gestação. Em relação ao número de gestações, 09 (52,9\%) eram primigestas, 05 $(29,4 \%)$ tiveram duas gestações, $02(11,8 \%)$ mais de três gestações e $01(5,9 \%)$ se encontrava na terceira gestação.

Quanto aos cuidados com o RN, a Tabela 1 mostra o conhecimento das gestantes quanto ao número de vezes que o bebê deve amamentar diariamente, 15 $(88,24 \%)$ gestantes consideram necessário amamentar em livre demanda $( \pm 8,1)$.

Tabela 1. Conhecimento das gestantes da Unidade de Saúde Santo Antônio em relação ao número de mamadas e necessidade de complementação à amamentação. Riacho dos Machados. 2015.

\begin{tabular}{llcc}
\hline \multicolumn{1}{c}{ Variável } & $\mathbf{n}$ & $\%$ & $\mathbf{\pm ( n )}$ \\
\hline Número de mamadas/dia & \multicolumn{4}{l}{} \\
\hline Livre demanda & 15 & 88,2 & \\
03 vezes & 01 & 5,9 & \\
08 vezes & 01 & 5,9 & \\
\multicolumn{4}{l}{ Necessidade de complementar a amamentação } \\
\hline Sim & 03 & 17,6 & \\
Não & 14 & 82,4 & 7,8 \\
\hline
\end{tabular}

Fonte: Dados de pesquisa. 2015.

Geralmente um bebê em aleitamento materno exclusivo, mama de oito a 12 vezes ao dia, mas não há restrições de horários e de tempo de permanência da criança na mama, sendo normal nos primeiros meses de vida, que a criança mame com frequência e sem horários regulares ${ }^{9}$. 
Observa-se que 14 (82,35\%) gestantes não consideram necessário complementar a amamentação do recém-nascido e 03 (17,65\%) afirmaram que é necessário complementar $( \pm 7,8)$ (Tabela 1).

A Organização Mundial da Saúde (OMS) e o Ministério da Saúde recomendam que o aleitamento materno seja mantido exclusivo nos primeiros seis meses de vida e associado a outros alimentos até dois anos ou mais. No entanto várias mães, especialmente, as que estão inseguras e as com baixa autoestima, costumam complementar precocemente e de forma desnecessária a amamentação do bebêt ${ }^{10}$.

Além de ser uma técnica simples e de baixo custo financeiro, o aleitamento materno é a melhor fonte de nutrientes necessários à saúde infantil e atua na imunidade da criança, protegendo-a de infecções, doenças respiratórias, alergias, diarreia, obesidade entre outras doenças ${ }^{11}$.

Sobre o tipo de alimento que pode ser complementado à amamentação do recém-nascido, as gestantes que afirmaram essa necessidade citaram alimentos como frutas, sopas, verduras e água como pode ser verificado nas respostas abaixo:

"Frutas, sopinha e verdura" (IJSS., 24 anos).

"Antes dos seis meses, no meu caso, eu daria água" (VCSS., 33 anos).

“É bom dá alguma fruta e água pra ajudar né." (G.M.S, 26 anos)

A amamentação da criança deve ser complementada com outros alimentos, a partir do sexto mês de vida. É recomendado introduzir os novos alimentos gradativamente, respeitando a evolução da criança, que deve receber três refeições complementar ao dia. Inicialmente deve ser introduzida às papas de fruta e papa salgada $^{12}$.

Em relação ao banho do bebê, as gestantes foram questionadas por onde devem iniciá-lo. Os dados da Tabela 2 apontam que 9 (52,9\%) consideram que o banho do bebê deve ser iniciado pela cabeça, seguido pelo rosto e corpo $( \pm 3,2)$. 
Tabela 2. Conhecimento das gestantes da Unidade de Saúde Santo Antônio em relação à superfície corporal para início do banho do bebê e higienização do coto umbilical. Riacho dos Machados. 2015.

\begin{tabular}{llll}
\hline \multicolumn{1}{c}{ Variável } & $\mathbf{n}$ & $\%$ & $\mathbf{\pm ( n )}$ \\
\hline Superfície corporal para início do banho & & & \\
\hline Cabeça seguida pelo rosto e corpo & 09 & 52,9 & \\
Rosto seguido pelo corpo e cabeça & 03 & 17,6 & \\
Corpo seguido pelo rosto e cabeça & 03 & 17,6 & \\
Não sabe & 02 & 11,9 & 3,2 \\
\hline Momento da higienização do coto umbilical & & \\
\hline Após cada troca de fralda e/ou banho & 04 & 23,6 & \\
Somente após o banho & 10 & 58,8 & \\
Não sabe & 03 & 17,6 & 3,8 \\
\hline
\end{tabular}

Fonte: Dados de pesquisa. 2015.

A pele do bebê é sensível, fina e frágil, por isso os produtos de higiene e proteção devem ser rigorosamente escolhidos, para preservar a integridade cutânea e diminuir os riscos provocados pelo cuidado indevido, evitando assim a diminuição da sensibilidade e defesa da pele do bebê contra a excessiva proliferação microbiana ${ }^{13}$.

Ao serem questionadas sobre quais os cuidados que se devem ter na hora do banho do bebê, as gestantes informaram cuidados com cabeça, ouvido, olhos, umbigo, temperatura da água e demonstraram dúvidas, medo e insegurança para essa atividade, conforme se verifica nos em discursos:

"Não deixar cair água no ouvido, no nariz, acho que é isso mesmo, sabão nos olhos, acho que é isso, se tiver mais coisa, eu não lembro" (GMS., 26 anos).

"[...] eu ainda tenho que aprender, né?, porque é o primeiro filho" (KTA., 24 anos). 
"Banhar em ambiente fechado, com a água na temperatura morninha, ter cuidado com ouvidinho, narizinho. Com o umbigo, se caso não tiver caído, ainda, é isso, eu

acho"

(DPS., 20 anos).

"Eu não banho quando tá novo, quem banha é minha irmã, minha mãe. Eu não tenho coragem, para explicar assim direitinho, eu não sei não. Eu não cuido quando

tá novinho, eu cuido quando cai o umbigo, eu tenho cuidado com a cabecinha, porque é muito sensível. Não tenho conhecimento, ninguém nunca me falou isso, no hospital" (LFS., 23 anos).

Muitas dificuldades são encontradas pelas mães na hora do banho do RN, especialmente as primíparas, podendo também acontecer com algumas multíparas, uma vez que o sentimento de medo ao prestar os cuidados, está ligado ao tamanho, a fragilidade do $\mathrm{RN}$ e a higienização do coto umbilical ${ }^{14}$.

Durante o banho há um excesso na manipulação do $\mathrm{RN}$, que pode provocar inúmeras reações. Esta situação é caracterizada pela interação entre o adulto e o bebê, onde sua reação está diretamente relacionada às manipulações e a adequação do procedimento. O banho é retratado como algo prazeroso para o RN, pois o ambiente líquido e quente lembra o útero materno ${ }^{15}$.

O banho do RN deve ser iniciado pelo rosto, sem sabão, faz-se limpeza dos olhos, seguindo para as narinas e orelhas. Dentro de uma bacia com água morna 0 bebê deve ser posicionado de modo que seu corpo fique submerso até o pescoço. $O$ ambiente deve ser fechado, a fim de evitar perdas de calor. Em seguida ensaboar o pescoço, membros superiores, tórax anterior, costas, membros inferiores e genitálias sucessivamente e retirar o sabonete. Retirar o RN da bacia, envolto em toalha macia e secar a pele com movimentos compressivos e suaves ${ }^{16}$.

Em relação à higienização do coto umbilical, a Tabela 2 mostra a opinião das gestantes sobre quando deve ser realizada a limpeza, entre as entrevistadas 10 $(58,8 \%)$ acreditam que a limpeza deve ser feita somente após o banho $( \pm 3,8)$. Notase que a maioria das gestantes se mostrou pouco orientada quanto os cuidados com o coto umbilical. 
O coto umbilical deve ser mantido limpo e seco, devendo ser realizada a limpeza uma vez ou mais ao dia, entre os produtos a serem utilizados no processo de limpeza, a clorexidina é eficaz na redução da colonização e infecção do coto e o álcool $70 \%$ acelera a mumificação e não interfere na colonização ${ }^{17}$.

No que diz respeito à posição que o bebê deve ser colocado na hora de dormir, os dados da Tabela 3 revelam que 9 (52,9\%) gestantes acreditam que 0 bebê deve ser colocado de barriga para cima (decúbito dorsal) $( \pm 3,2)$.

Tabela 3. Conhecimento das gestantes da Unidade de Saúde Santo Antônio em relação à posição para o bebê dormir. Riacho dos Machados. 2015.

\begin{tabular}{lccc}
\hline \multicolumn{1}{c}{ Variável } & $\mathbf{n}$ & $\%$ & $\mathbf{\pm ( n )}$ \\
\hline Posição para o bebê dormir & & & \\
\hline Decúbito dorsal & 09 & 52,9 & \\
Decúbito lateral & 07 & 41,2 & \\
Não sabe & 01 & 5,9 & 3,2 \\
\hline
\end{tabular}

Fonte: Dados de pesquisa. 2015.

Esses dados são discordantes dos encontrados em um estudo realizado com puérperas da Santa Casa de Misericórdia e no Hospital Universitário da Universidade Federal do Rio Grande, ao avaliar, após uma campanha nacional, o conhecimento materno sobre posição do bebê na hora de dormir, identificaram que a maioria das mães $(76,8 \%)$ consideram que a posição que o bebê deve assumir para dormir é de lado, enquanto $20 \%$ responderam que é de barriga para cima, $1 \%$ de barriga para baixo e 2,2\% não sabem qual a melhor posição para o bebê dormir ${ }^{18}$.

Em relação à higiene oral do bebê, a maioria das entrevistadas (14: 82,4\%) declarou não saber como e quando dever ser realizada, como pode ser observado nas falas abaixo:

"Não sei, eu imagino, com o lenço, algodão úmido [...]" (IJSS., 24 anos).

"Não sei quando. Não sei como é realizada" (DBX., 26 anos). 
“Não sei não, recém-nascido, eu não sei não. Eu nunca ouvi falar" (SMF., 31 anos).

"Criança recém-nascida eu não limpo não, só quando ta nascendo o dentinho" (JMB., 28 anos).

"Eu não lembro disso não, novinho? Eu limpava quando tava sujo, por fora, sempre depois que da peito, eu não limpava por dentro não[...]" (LFS., 23 anos).

É necessário que os pais se conscientizem dos cuidados constantes de higienização com a boca do bebê, fazendo com que desde o nascimento o bebê já tenha saúde bucal, e para isso a higienização deve ser feita com uma gaze ou ponta de fralda ou ainda uma dedeira de tecido, seco ou embebido em água filtrada, fervida ou soro fisiológico ${ }^{19}$.

No que diz respeito à posição que o bebê deve ser colocado para arrotar após a amamentação, os discursos das entrevistadas revelam que têm conhecimento, como pode ser observado nos trechos citados abaixo:

"Em pé, encostado no peito" (VCSS., 33 anos).

"Em pé, deitadinho no ombro" (LFS., 23 anos).

"Em pezinho, virado as costas para mim" (FKFS., 28 anos).

"Em pé, a gente apoiando e segurando a cabeça próximo ao ombro" (IJSS., 24 anos).

"Em pé, no ombro, deitadinho no ombro, com a cabecinha virada de forma que ele possa arrotar" (KAA., 26 anos). 
Ao terminar de mamar, o bebê deve ser colocado para arrotar, a posição ideal é vertical com a cabeça apoiada sobre o ombro da mãe. Essa técnica evita que o RN broncoaspire leite regurgitado ${ }^{20}$.

\section{CONSIDERAÇÕES FINAIS}

Percebeu-se que algumas não têm um conhecimento suficiente acerca dos cuidados com o RN, quanto à higiene oral, banho, e cuidados com o coto umbilical, o que leva a um despreparo que pode comprometer o desenvolvimento saudável da criança, uma vez que o reconhecimento e a interpretação correta da mãe em relação aos sinais que o RN emite é essencial para manter a sua saúde e o seu bem-estar.

Um fator importante e que pode influenciar na insuficiência do conhecimento das gestantes acerca dos cuidados com o RN, é que a maior parte das mulheres eram primigestas e consequente não têm a experiência e segurança que são esperadas nas multíparas. Outro fator a ser considerado é que muitas mulheres passam a responsabilidade dos cuidados com o RN para familiares, fugindo do seu dever de mãe em obter conhecimentos para cuidar de seu filho.

\section{REFERÊNCIAS}

1. Brasil. Ministério da Saúde. Secretaria de Atenção à Saúde. Departamento de Atenção Básica. Atenção ao pré-natal de baixo risco. - Brasília : Editora do Ministério da Saúde, Cadernos de Atenção Básica, n 32. 2012. 318 p.

2. Brasil. Ministério da Saúde. Secretaria de Atenção à Saúde. Departamento de Atenção Básica. Atenção ao pré-natal de baixo risco [recurso eletrônico]. - 1. ed. rev. - Brasília : Editora do Ministério da Saúde, Cadernos de Atenção Básica, n 32. 2013. 318 p. [acesso: 19 jun. 2015]. Disponível em: http://189.28.128.100/dab/docs/portaldab/publicacoes/caderno 32.pdf.

3. São Paulo. Secretaria da Saúde. Coordenadoria de Planejamento em Saúde. Assessoria Técnica em Saúde da Mulher. Atenção à gestante e à puérpera no 
SUS - SP: manual técnico do pré-natal e puerpério. - São Paulo: SES/SP, 2010. $234 \mathrm{p}$.

4. Barreto CNB, Ressel LB, Santos CC dos, Wilhelm LA, Silva SC da, Alves CN. Prenatal Care in the Voice of Pregnant Women. J Nurs UFPE on line., Recife, 2013; 7(6): 4354-63, jun. [acesso: 02 mai. 2015]. Disponível em: http://dx.doi.org/10.5205/reuol.4164-33013-1-SM.0706201306.

5. Ribeiro JZB. Importância das orientações no pré-natal: conhecendo a visão das puérperas. [monografia]. Graduação em Enfermagem. Pelotas: Universidade Federal de Pelotas, 2011. 54 p.

6. Zani AV, Tonete VLP, Parada CG de L. Care For Low Weight Newborn Infants By Family Health Teams: Integrative Review. Rev enferm UFPE on line., Recife, 2014; 8(5): 1347-56, mai. [acesso: 08 jun. 2015]. Disponível em: http://dx.doi.org/10.5205/reuol.5863-50531-1ED.0805201432.

7. Bergamaschi SFF, Praça NS. The Adolescent puerperae's experience of taking care of the newborn at home. Rev. esc. enferm. USP [online]. São Paulo, 2008; 42(3): 451-457, set. [acesso: 23 mai. 2015]. Disponível em: http://dx.doi.org/10.1590/S0080-62342008000300006.

8. Malaquias $T$ da SM, Gaiva MAM, Higarashi IH. Perceptions of the family members of children regarding well-child check-ups in the family healthcare strategy. Rev. Gaúcha Enferm. [online]. 2015; 36(1): 62-68, jan./mar. [acesso: 25 jul. 2015]. Disponível em: http://dx.doi.org/10.1590/1983-1447.2015.01.46907.

9. Brasil. Ministério da Saúde. Secretaria de Atenção à Saúde. Departamento de Atenção Básica. Saúde da criança: nutrição infantil: aleitamento materno e alimentação complementar. - Brasília : Editora do Ministério da Saúde, Cadernos de Atenção Básica, n. 23. 2009. 112 p. 
10. Brasil. Ministério da Saúde. Secretaria de Atenção à Saúde. Departamento de Atenção Básica. Saúde da criança : aleitamento materno e alimentação complementar. - 2. ed. - Brasília : Ministério da Saúde, Cadernos de Atenção Básica n. 23, 2015. 184 p.

11. Pereira ALT. Os benefícios da amamentação. [dissertação]. Programa de Pósgraduação Scritu Sensu em Medicina Dentária. Universidade Fernando Pessoa. Faculdade de Ciências da Saúde. 2013. 73 p.

12. Minharro $M C$ de $O$. Tendência e determinantes das práticas de alimentação complementar em crianças menores de 12 meses no município de Botucatu-sp: 2006 e 2010. [dissertação]. Programa de Pós-Graduação em Enfermagem Mestrado Profissional. Faculdade de Medicina de Botucatu da Universidade Estadual Paulista Julio de Mesquita Filho. 2012. 92 p.

13. Fernandes DJ, Machado MCR, Oliveira ZNP. Children and newborn skin care and prevention. An Bras Dermatol. 2011; 86(1): 102-10, jan./feb. [acesso: 17 mai. 2015]. Disponível em: http://dx.doi.org/10.1590/S036505962011000100014.

14. Morais AC, Campos C dos S de C. Cuidando do Filho Recém-Nascido: Vivência de Adolescentes Primíparas. Rev enferm UFPE on line. 2011; 5(10): 2406-14, dez. [acesso: 13 mar. 2015]. Disponível em: http://dx.doi.org/10.5205/reuol.213315571-1-LE.0510201110.

15. Brasil. Ministério da Saúde. Secretaria de Atenção à Saúde. Departamento de Ações Programáticas Estratégicas. Atenção humanizada ao recém-nascido de baixo peso: Método Canguru. - 2. ed. - Brasília : Editora do Ministério da Saúde, 2011. $204 \mathrm{p}$. 
16. Brasil. Ministério da Saúde. Secretaria de Atenção à Saúde. Departamento de Ações Programáticas e Estratégicas. Atenção à saúde do recém-nascido: guia para os profissionais de saúde. - Brasília : Ministério da Saúde, 2011. 192 p.

17. Brasil. Ministério da Saúde. Secretaria de Atenção à Saúde. Departamento de Ações Programáticas Estratégicas. Atenção à saúde do recém-nascido : guia para os profissionais de saúde. - 2. ed. - Brasília : Ministério da Saúde, 2012. $195 \mathrm{p}$.

18. Cesar JA, Cunha CF, Sutil AT, Santos GB dos S. Opinião das mães sobre a posição do bebê dormir após campanha nacional: estudo de base populacional no extremo sul do Brasil. Rev. Bras. Saúde Mater. Recife, 2013; 13(4): 329333, out./dez. [acesso: 17 mai 2015]. Disponível em: http://dx.doi.org/10.1590/S1519-38292013000400005.

19. Rocha SF, Pires AL. Odontologia na Primeira Infância. In: I Jornada Odontológica UNIP- Goiânia. Anais da Jornada Odontológica UNIP Goiânia, 21 a 22 de out. de 2009. Goiânia - GO. p. 79.

20. Leonart, E. Cuidador Infantil. 1 ed. Paraná, Editora IFPR- Instituto Federal do Paraná, 2012. 158 p. [acesso: 18 mai. 2015]. Disponível em: http://200.17.98.44/pronatec/wp-content/uploads/2012/07/cinf1.pdf. 